\title{
Simulation of pump performance control schemes with the use of the virtual model of the process
}

\author{
Julia Nikolaevna Zatsarinnaya ${ }^{1, *}$, Nataliya Alexandrovna Staroverova $^{2}$, Alla Grigorevna Logacheva $^{1}$ \\ ${ }^{1}$ Kazan Power State University, Kazan, Russian Federation \\ ${ }^{2}$ Kazan National Research Technological University, Kazan, Russian Federation
}

\begin{abstract}
The research of pump performance control schemes based on bypassing depending on the moving medium is carried out with the use of the virtual model of the process. Such approach speeds up and simplifies the development and reduces the risk of errors made by the developer in the execution of routine operations. Unlike theoretical methods of research, the virtual model of the process allows the developer to concentrate on the physics of simulated actions or the principles of the functioning of technological systems, without delving into the subtleties of programming. It is established that this simulator will significantly improve the operator's work, provide high-quality process control, and will also allow to ensure safe operation in the real conditions in the chemical-technological production.
\end{abstract}

\section{Current state of the issue}

In the modern world, energy saving issues are closely related to the development of the world economy and in the last decade are becoming more relevant for the Russian economy. Most large-scale productions in Russia, such as the oil and chemical industry, thermal power plants and petrochemical industries were built in the former USSR before the 1980s. The Soviet industry can be characterized by the growth of capacity per unit of aggregates, the growth of oil and gas production and the constantly growing demand for electricity. This led to the construction of powerful oil arteries and large power plants. Despite this, the main problem in the operation of oil pipelines up to the 1980s was the search for a reserve to increase the throughput capacity of main oil pipelines. In the mid-1980s, there was a tendency to decline in oil production, but in general, until 1990, the growth in pumping volumes still remained. By the end of the 1990s, due to political and economic instability in Russia, oil pumping volumes in some pipelines declined by $50 \%$. Nowadays, oil pumping volumes have increased by $30 \%$ compared to the $90 \mathrm{~s}$, but the reduction in pumping volumes below the nominal pumping capacity has led inefficient pump operating [1].

The aim of our work was modeling of pump performance control schemes and increasing the efficiency of main oil pipelines, using a virtual model of the process, which results in energy savings in the operation of pumps in the underload condition. This model can be used for modeling the operating modes of smoke exhausters and fan blowers of boilers, oilpumping pumps and gas-pumping compressors.

In recent years intensive work is being done on experimental and theoretical studies of the operation of pumping stations in variable modes and methods of control of their operation in such modes by a number of authors such as A.A Veremeenko, E.V. Vinogradova, E.V. Vyazunova, A.I. Golyanova, L.A. Zaitseva, L.G. Kolpakova, Sh.I. Rakhmatullina, N.Z. Aitova, G.S. Salashchenko, In works under consideration, three most common technical schemes for pumps operation control are noted: the control of the rotor speed of the pump, using variable-speed motors; the control of the rotor speed of the pump, using special control couplings; change of the geometry of the flow channels of the pump and the kinematics of the flow at the input to the impeller.

However, some of the technical solutions listed above have not been applied for a number of reasons: the lack of equipment manufactured in Russia and that would comply with requirements for energy-intensive pumping and power equipment and the lack of fundamental research in this area. Conducting of the experiment was restrained because of the lack of mathematical models of the elements of the system, adequately describing their characteristics. We conducted a comparative study of pump performance control schemes based on bypassing and throttling, depending on the moving medium by the virtual process modeling. Using of the virtual model of the process speeds up and simplifies development, lowers the risk of mistakes made by the developer in the execution of routine operations, allows the developer to concentrate on the physics of simulated actions or the principles of the functioning of technological systems, without delving into the subtleties of programming.

The following main research tasks can be distinguished:

\footnotetext{
* Corresponding author: waysubbota@gmail.com
} 
- Development of mathematical (virtual) models of the process of automatic control of pump performance based on stabilization by throttling on the basis of modern technical automation tools using the Visual Modeler modeling tool.

- Development of an algorithm for calculating the operation modes of the pump and minimizing the energy consumption.

\section{Review of modern tools for dynamic modeling}

The environments for the technological process simulators development are considered. The following systems were chosen for analysis and comparison:

The CENTUM VP system represents a distributed production control system used to manage and control the operation of plants in a wide variety of industries: oil refining, petrochemical, chemical, pulp and paper, and other industries. It consists of the following components:

- Operator station (HIS) is the operator interface designed for control, monitoring and engineering;

- Control Station (FCS) is a controller that performs automated real-time monitoring and control of the technological process. It allows to perform the following actions:

- Define input / output modules, create switches (Switch);

- Implement logic control schemes using five programming languages.

- Vnet / IP network. All Centum VP system control units are connected via the Vnet / IP bus. It provides data transfer between control stations and operator stations. This bus is a real-time bus and all its traffic is controlled by the system. To connect the operator station to the Vnet / IP network the control bus interface board VI701 is used.

The Trace Mode system is a software package of SCADA HMI class, invented by AdAstra Research Group. It is specialized for the development of software for automated process control systems, telemechanics systems, building automation, systems for electricity, water, gas, heat accounting, and for control of their operation in the present time [2].

A distinctive feature of TRACE MODE is "the technology of a single line programming", that is, the probability of developing all the ACS modules with the support of a single device. The development of a single line programming allows creating human-machine interface tools, resource accounting systems, program industrial controllers and develop a web-interface within the framework of one project. For this reasons special editors are integrated into the TRACE MODE tool system, such as:

- Editor of graphic mnemonic schemes;

- The editor of the screen panels;

- The editor of programs in visual language FBD (standard IEC 6-1131/3);

- The editor of programs in visual language SFC (standard IEC 6-1131/3);
- The editor of programs in visual language LD (standard IEC 6-1131/3);

- The program editor in the procedural language ST (IEC standard 6-1131/3);

- Program editor in procedural language IL (IEC standard 6-1131/3);

- Document templates editor;

- Builder of links with DBMS;

- Equipment Passport Editor (EAM);

- Staff Editor (HRM);

- The editor of material resources (MES).

The CodeSys system is an instrumental software package for industrial automation. It is developed and distributed by 3S-Smart Software Solutions $\mathrm{GmbH}$ (Kempten, Germany).

The CoDeSys programming environment includes a set of tools for preparing and debugging programs, compilers, configurators, visualization editors, etc. If necessary, the functionality of the system is supplemented with optional components. The projects created in CoDeSys can be saved not only on the PC disk, but also in the controller, if it has a large memory size, what allows to avoid the loss of source code or dislocation in projects. For large projects, a version control system (SVN) is envisaged.

In CoDeSys all 5 programming languages defined by the IEC 61131-3 standard are available:

- IL (Instruction List) - assembler-like language;

- ST (Structured Text) - Pascal-like language;

- LD (Ladder Diagram) - Relay-like schemes;

- FBD (Function Block Diagram) - Multi-functional block language;

- SFC (Sequential Function Chart) - The language of state diagrams.

The InSIDE system is a built-in development environment for InSiDE simulators. It runs under the Windows family of operating systems (Windows NT, Windows 2000, Windows XP) and allows the creation of simulators (both analytical and full-scale) for technological objects of various complexity. It allows:

- Automation of the simulators development process, which not only speeds up and simplifies the development, but also reduces the risk of errors made by the developer in the execution of routine operations.

- Involvement in the development process of the engineers who are not professionals in programming languages. Applying CAD systems based on the principle of visual programming, the developer can focus on the physics of simulated actions or the principles of the functioning of technological systems, without delving into the subtleties of programming.

- Recording of the configurations in the simulator by user without involving developers.

Main features of the InSiDE:

- Graphical interface for the development of simulators;

- Multiuser development environment;

- CAD applications for visual programming;

- Tools for debugging and monitoring the work of the simulator;

- Automated integration of simulation modules; 
- The possibility of using distributed computing (cluster simulators).

The built-in development environment of InSiDE simulators is based on the application of the present-day database InterBase. The simulator database is used to store data according to the simulator being developed and to organize multi-user access to it.

The MATHLAB (MATrix LABoratory) software package is designed for performing scientific and engineering calculations, with the help of which the problems of mathematical modeling are effectively solved. The integrated development environment includes a set of development tools provided through a single user interface.

The Lab VIEW software package is a graphical programming environment used around the world for the rapid creation of complex applications in measurement and automation tasks based on the architecture of data flows.

The Omega Land system represents an integrated environment for dynamic modeling. It contains several "modules", each of which provides an independent function and a mechanism for the systematized connection of these modules (VMspace).

Basic functional modules include the necessary fundamental modules for constructing almost any application system. Optional function modules can be selected depending on the application. Both basic and optional function modules are developed by Omega Simulation Company. The basic function modules consist of the following four modules:

1. The module of the technological installation model (VisualModeler)

2. Execution Control Module (EXEC)

3. Database module (DataBase)

4. Graphic Builder

Each module provides various functions, which provides the flexibility of the system, which allows to model and solve any problems.

Using the "Visual Modeler" software package for dynamic modeling in combination with various modules, it is possible to create a system designed for process design / analysis, for checking control systems, for training, for operating support and so on. This system uses the following simulators:

- A static simulator can quickly model a stable state of equilibrium for certain process conditions. It is used in the following types of situations:

- Analysis of physical properties of chemical components;

- Analysis of design data and border conditions of each block;

- Definition of a steady mode of operation;

- Evaluation of construction and operation costs.

- Dynamic simulator allows to view the change in results depending on the design data provided for each unit, and also depending on the manipulations with the valves, etc. in the process of modeling. It is used in the following situations:

- Checking the start and stop operations;

- Analysis of the limit points of operations;

- Development and research of control systems;
- Analysis of accidents and contingencies.

The aim of both static and dynamic simulators is to get an idea of the process, and also to analyze the operating conditions. Nevertheless, since the main objective of the first one is to extract the design data for the equipment, and for the latter it is to analyze and optimize the operating conditions, it is important to make the right choice in accordance with the purpose of the analysis.

Visual Modeler contains numerous built-in functions, including the function of model editing, which provides the possibility of creating a simulator that works with various mathematical models of processes and devices. The package also includes programs that implement the functions of performing production operations used for the operation of developed simulator and its testing, as well as other functions that technologists and automation specialists may need to create special models for specific tasks.

Visual Modeler offers five standard functions and two libraries:

- Model editing function - this function is used to build a model of petrochemical production, which is the object of modeling, and to create a program that performs calculations;

- Model execution function - this function is used to control the running of model;

- Function of a block model development - this function generates a model of a new block and registers it in the library of unit models;

- Function for calculating of physical properties [S] [C] - The physical property data necessary for the model calculating contains such types of variables as molar mass, equilibrium gas-liquid flow ratio, etc.

Despite the fact that Visual Modeler represents a modular type of simulator, the balance of the pressure stream is not calculated separately for each block, but simultaneously for the whole process model [3].

Visual Modeler breaks the process topology into several "flow lines" for each execution cycle. The flow rate remains the same throughout the single flow line.

As a result of the analysis, it was concluded that Visual Modeler is ideally suited for implementing the process of controlling the operation of the pump. Since this environment has many advantages in comparison with similar systems for the development of visual simulators.

\section{Description of the proposed solution}

When implementing a process diagram, it is necessary to add a system (in other words, to describe for which mediums the circuit will be used). Since this project provides the implementation of three processes, three systems have been added.

To add a system, the components and their physicochemical properties are determined. There is a large database of component in the VM, but even if the required component is not available, it is possible to create and describe it. In our case, for the initial settings, the available components can be used. 
Once the systems have been added, it is possible to implement the process scheme itself. On the next step, the settings and process sensors are configured.

Using a database of existing equipment, circuits were drawn up, pumps, valves, regulators, and measuring instruments were selected.

The constructed scheme made it possible to carry out research in several directions: 1 . studying the behavior of various systems on the equipment used, and how to anticipate possible difficulties in introducing new components or new equipment into those processes. 2 . Training of personnel and prevention of emergencies through their modeling. 3. Selection of optimal parameters and control schemes to improve process performance.

\section{Conclusions}

The result of modeling and conducted research is the solution to the problem of rational loading of main oil pipeline systems. The main part of the research carried out includes complexes of mathematical algorithms for solving the problems of rational operation of main oil pipeline systems and improving the management efficiency of main oil pipelines. This task was solved on the basis of creating a simulator system for automatic regulation of the pump efficiency based on the stabilization method by throttling with the Visual Modeler modeling tool.

As a result of creating of a virtual model of the pump performance control scheme, it became possible to increase the efficiency of the main oil pipelines, resulting in energy savings in the operation of pumps in the underload condition by $12-15 \%$.

The proposed algorithms of the mathematical model make it easier to understand the process of pumps control, enabling future specialists to learn how to manage processes directly before starting working at the site. The use of the developed model and algorithms allowed to cut energy costs on the Surgut-Polotsk and Baku-Tikhoretsk pipelines to 5.5 and $7 \%$, respectively, when solving problems of rational operation and transportation of oil.

\section{References}

1. A.A. Kabanov, S.A. Dubovik. Problemy upravleniya i informatiki, 3, 17 (2010)

2. B.T. Polyak, E.N. Gryazina, A.A. Tremba. Avtomatika i telemekhanika, 12, 3 (2008)

3. M.V. Astakhov, T.V. Tagantsev. Proc. of the Bauman MSTU "Mathematical Modeling of Complex Technical Systems", 593, 125 (2006) 